\title{
Definition of form and Parameters of quantum of space of the Universe
}

\author{
Valentyn Alexeevich Nastasenko*
}

Professor, Department of Transport Technologies, Kherson State Maritime Academy, 141, Ushakovast. 41, 73003, Kherson, Ukraine

\section{Article Info}

\section{*Corresponding author: Valentyn Alexeevich Nastasenko \\ Professor \\ Department of Transport Technologies \\ Kherson State Maritime Academy \\ 141, Ushakovast. 41, 73003 \\ Kherson, Ukraine \\ Tel: +38(050) 807-91-99 \\ E-mail: nastasenko2004@ukr.net}

\section{Received: January 21, 2019 \\ Accepted: January 29, 2019 \\ Published: February 4, 2019}

Citation: Nastasenko VA. Definition of form and Parameters of quantum of space of the Universe. Int J Cosmol Astron Astrophys. 2019; 1(2): 42-45.

doi: 10.18689/ijcaa-1000111

Copyright: (c) 2019 The Author(s). This work is licensed under a Creative Commons Attribution 4.0 International License, which permits unrestricted use, distribution, and reproduction in any medium, provided the original work is properly cited.

Published by Madridge Publishers

\begin{abstract}
In this work the substantiation of form and parameters of a quantum space of the Universe is given. It is shown that solution of this problem has level of scientific discovery, since reliable information about it is unknown, except for its general characteristicsquantum it should be minimum and indivisible. This problem is traced to definition of fundamentals of material world structure and parental elements for its construction, that were earlier considered to be atoms, and then-elementary particles. However, it has not been solved full and complete till present time. Whereas, the level of development of knowledge of material world and the Universe fundamentals depends on it to a large extent, that is why its solvation is an important and highly topical problem for the development of the contemporary science. It has especially great significance for philosophy development, quantum physics, physics of elementary particles and cosmology. It is shown that presence of wave properties of elementary particles permits to connect quantum of space with wave parameters of gravitational field, which have wave frequency $v_{G}=7.4 \cdot 10^{42} \mathrm{~Hz}$ and wave length $\lambda_{G}=c / v_{G}=4.051249|432| \cdot 10^{-35} \mathrm{~m}$. This connection creates evident physical meaning to quantum of space parameters and possibility of strict problem solvation of their numerical definition. Quantum form was also identified, on and make to as hexagonal prism, which attains tight packing of quant's in the Universe.
\end{abstract}

Keywords: Quantum of the Universe space; Elementary particles; Cosmology; Parameters of Gravitational field.

\section{Introduction}

Argumentation of quantum of space parameters of the Universe is a complicated scientific problem, as its reliable information is unknown, except for its general characteristics-quant should be minimum and indivisible. Actually, this problem is traced to definition of fundamentals of material world structure and parental elements "bricks" for its construction, that were earlier considered to be atoms, and thenelementary particles. Its solution had and still has a great significance for the majority of top-level world scientists throughout history of civilization [1]. However, it has not been solved full and complete till present time. Whereas, the level of development of knowledge of material world and the Universe fundamentals depends on it to a large extent, that is why its solution is an important and highly topical problem for the development of the contemporary science. It has especially great significance for philosophy development, quantum physics, physics of elementary particles and cosmology. Solvation of the given problem constitutes the main aim of the executed work. Strict argumentation and definition of possible real shape and parameters of quantum of the Universe space, based on the indubitable principles of physics, constitute its scientific novelty. 


\section{Analysis of problem state, problem formulation and search of the lines of its solution}

In the framework of general principles solution of scientific-technical problems [2], two original sub problems should be outlined:

A - definition of quantum of space form;

$B$ - definition of quantum of space parameters.

Traditional concept of form $A$ in the shape of balls, that has a minimum diameter $D_{\text {min' }}$ is unacceptable, as the quanta of space should be tightly packed, and balls leave empty space in between. A small ball with diameter $d$, that is lesser than diameter $D_{\text {min }}$ of parental small balls, can be inscribed into this space (Figure 1). A property of initial balls to form free spaces that are much smaller than quantum of space, created by these balls, when contacting each other, contradicts the principle of quantum minimalist reaching.

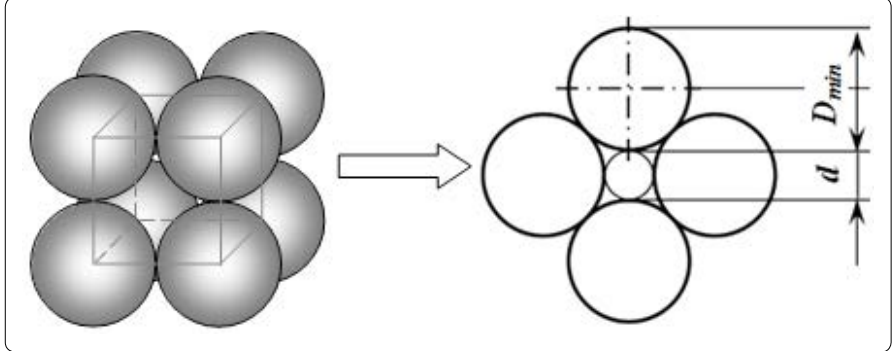

Figure 1. Grouping of quantum of space of spherical shape

Parameters $B$ are also difficult to be defined as the quantum of space should be the minimal possible value in the Universe, and its limits. These limits and the possibility of these limits existence itself have not been well grounded. It is explained by suggested by philosophers a hypothesis in the $20^{\text {th }}$ century about the infinite reduction opportunities of material world objects in the frameworks of inexhaustibility of structure electron [3].

Thus, the absence of known, or alike input information and its transformation rules into the ultimate one, converts this task in hand to the $5^{\text {th }}$ level of complexity, connected with scientific discoveries [2,4]. Procedures of search of such kind of solutions are indistinct and undetermined, thus they hardly yield to formal characterization. However, a total absence of rules and information for scientific discoveries finding do not exist as the main starting point is consilience of this search to known laws of nature, logicality of scientific knowledge development from simple to complicated, and also fundamentals of material world structure.

\section{Analysis of known solutions}

In a piece of work performed earlier [5] which is little known, provision has been made to the conclusion that the tight packing of not compressible and indivisible quantum particles is contributed by the elementary scheme, shown in (Figure 2). On the ground of thermodynamics conditions, connected with minimization of heat transfer, formation of tightly packed structure makes the most sense in the shape of regular hexagonal prisms, for which a central quantum is identical to the rest of quant's.

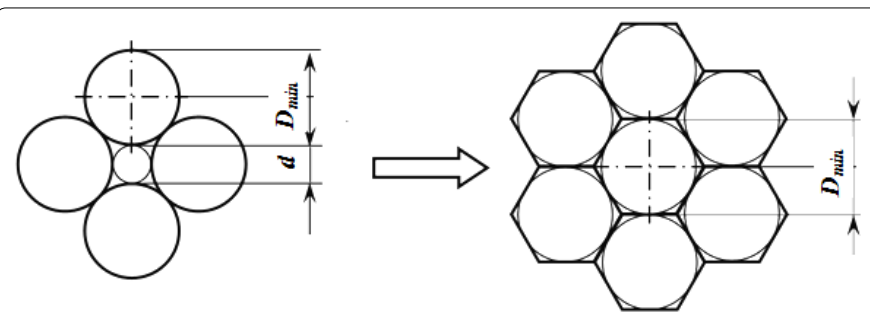

Figure 2. Transformation of spherical shape figures into a tightly packed hexagonal prism with $g$ transformation of central fragment

However, form of hexagonal quanta of space, shown in figure 2 , leads to binding of minimum length $\lambda_{G}$ to circumference diameter $D_{\min ^{\prime}}$ inscribed of a hexaeder. In this connection, a contradiction emerges, as with the diameter $D_{\min }$ of the inscribed circumference the sizes $\lambda_{G 6}$ of hexagonal lateral faces do not quantize (2) that follows from functional connection (1):

$\lambda_{G 6}=D_{\min } \operatorname{tg} 30^{\circ} ;(1)$

$\frac{D_{\min }}{\lambda_{G 6}}=\frac{1}{\operatorname{tg} 30^{\circ}}=\frac{1}{0.5773502692}=1.732050808 \neq 2$.

Hexagonal structure, shown in figure 3 , whose minimal size $\lambda_{G}$ is connected with lateral face, and its value is strictly quantized with the diameter $D_{\text {min }}$. of inscribed circumference in a hexaeder, lets to remove the stated defects:

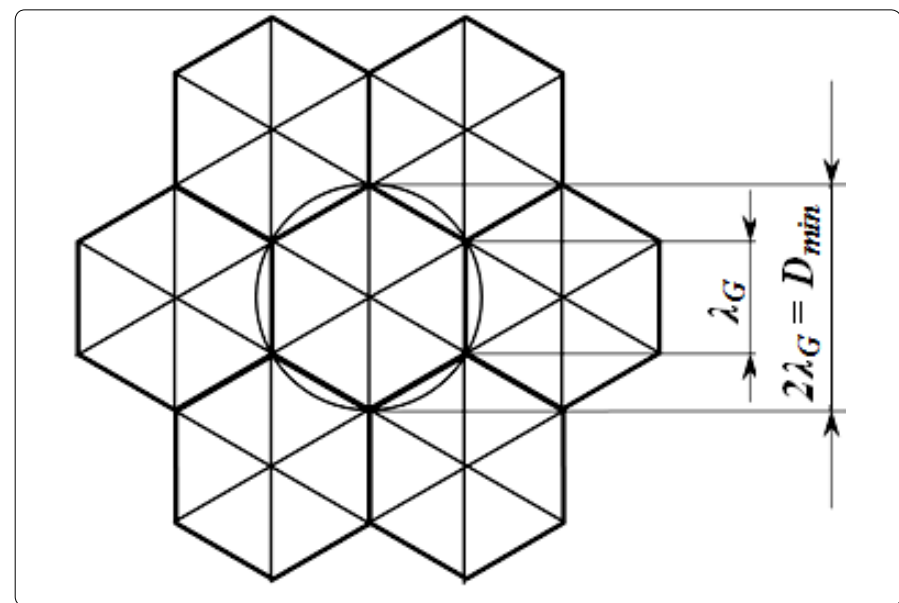

Figure 3. Rigorous form of tightly packed quantum structure of the Universe

The further move of problem solving of tight packing of quanta of the Universe space has shown that replacing of quanta of space of ball shape form by quanta of cubi form contributes it in the simplest way.

However, the square in its base doesn't comply with the minimal principle, as the simplest form is a triangle, which with the form of quarks connected. Thus, cube should be substituted by a straight-sided trihedral prism that is accepted as parental constituent element of quantum of the Universe space (hereinafter referred to as elementary, or the simplest quantum).

Circular grouping of 6 such kind of prisms, contiguous to each other by lateral faces with common central axes, forms complete quantum of space of the Universe in the shape of hexagonal prism (hereinafter referred to as cyclic quantum). Gravitational radius binds constituent elementary quanta into 
a circular quantum. Altitude of prisms is also equal to quantum value $\lambda_{G}$. In the frameworks of found solution, quantum of space of the Universe is as follows (Figure 4).

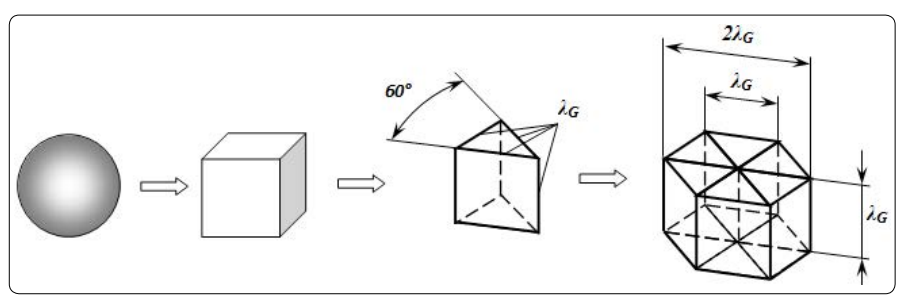

Figure 4. Concept of quantum of space of the Universe

Peculiarity of elementary and circular quanta of space figure 4 is that motion inside them is possible with quantum jumps along ribs only-communication lines of junctures of figures created by them. This is because of the general principles of quantization-constant value of minimal jump at change of any parameter. That is why motion along square sides or altitudes of triangle bases is impossible, they just do not exist, as divisibility of sizes to original sizes of ribs, which have minimal quantum value, is not observed for them.

In previous work [5] at this stage problem A considered as settled.

When solving problem $B$, in previous work [5] used Planck's values: long $l_{p}(3)$, time $t_{p}(4)$ and mass $m_{p}(5)$, which are derived from dependency from given in work [6]:

$l_{p}=\sqrt{\frac{h G}{c^{3}}}=\sqrt{\frac{6.62607004 \cdot 10^{-34}\left(\frac{\mathrm{kg} \cdot \mathrm{m}^{2}}{\mathrm{~s}}\right) \cdot 6.67408 \cdot 10^{-11}\left(\frac{\mathrm{m}^{3}}{\mathrm{~kg} \cdot \mathrm{s}^{2}}\right)}{\left[0.299792458 \cdot 10^{9}\left(\frac{\mathrm{m}}{\mathrm{s}}\right)\right]^{3}}}=4.05128 \cdot 10^{-35}(\mathrm{~m})$,

$t_{p}=\sqrt{\frac{h G}{c^{5}}}=\sqrt{\frac{6.62607004 \cdot 10^{-34}\left(\frac{\mathrm{kg} \cdot \mathrm{m}^{2}}{\mathrm{~s}}\right) \cdot 6.67408 \cdot 10^{-11}\left(\frac{\mathrm{m}^{3}}{\mathrm{~kg} \cdot \mathrm{s}^{2}}\right)}{\left[0.299792458 \cdot 10^{9}\left(\frac{\mathrm{m}}{\mathrm{s}}\right)\right]^{5}}}=13.5136 \cdot 10^{-44}(\mathrm{~s})$,

$m_{p}=\sqrt{\frac{h c}{G}}=\sqrt{\frac{6.626070040 \cdot 10^{-34}\left(\frac{\mathrm{kg} \cdot \mathrm{m}^{2}}{\mathrm{~s}}\right) \cdot 0.299792458 \cdot 10^{9}\left(\frac{\mathrm{m}}{\mathrm{s}}\right)}{6.67408 \cdot 10^{-11}\left(\frac{\mathrm{m}^{3}}{\mathrm{~kg} \cdot \mathrm{s}^{2}}\right)}}=5.45560 \cdot 10^{-8}(\mathrm{~kg})$.

Where $G$ - gravitational constant: $G=6.67408(31) \cdot 10^{-11} \frac{\mathrm{m}^{3}}{\mathrm{~kg} \cdot \mathrm{s}^{2}}$; $c$ - speed of light in vacuum: $c=0.299792458$ (exactly) $\cdot 10^{9} \frac{\mathrm{m}}{\mathrm{s}}$; h- Planck'sconstant:

$h=6.626070040(81) \cdot 10^{-34} \mathrm{~J} \cdot \mathrm{s}=6.26070040(81) \cdot 10^{-34} \frac{\mathrm{kg} \cdot \mathrm{m}^{2}}{\mathrm{~s}}$.

Magnitude and accuracy of parameters $G, c, h$ is recommended CODATA [7].

In previous work [5] at this stage problem B considered as settled.

However, M. Planck found parameters $l_{p^{\prime}} t_{p^{\prime}} m_{p}$ strictly mathematically, wishing to obtain on the basis of constants $G$, $c$, $h$, values with dimensions meter $(\mathrm{m})$, second (s), kilogram $(\mathrm{kg})$, which have not real analogies in the University. That is why Planck's parameters (3)..(5) make no physical sense, and also contradict harmony of masses and size that have developed in the material world. For example, comparing them with the dimensions and mass characteristics of an electron gives mutually opposite discrepancies: in size -
Planck's length $l_{p}=4.05128 \times 10^{-35} \mathrm{~m}$, they are $10^{20}$ times smaller classical electron radius $r_{e}=2.8179409 \times 10^{-15} \mathrm{~m}$; however Planck's mass $m_{p}=5.45560 \times 10^{-8} \mathrm{~kg}$ - they are $10^{23}$ times bigger electron rest mass $m_{e}=9.1093897 \times 10^{-31} \mathrm{~kg}$.

Analysis of the found parameters of elementary quanta and circular quantum of space has shown that they require more precise definition, that is performed in the given work.

\section{Argumentation of the new parameters of quantum of space of the Universe}

The difference of this work is used the author's earlier scientific discovery $[8,9]$ was considered - the strict definition of the gravitational field waves frequency $v_{G} \rightarrow 7.4 \cdot 10^{42} \mathrm{~Hz}$ (it's constant Nastasenko). Connection of further found quantum parameters with a gravitational field gives them pure physical meaning and makes alleviate the problem solvation of their numerical definition, as value $v_{G}$ is proved by functional connection (6). That strictly follows from found in the pieces of works [8,9] physical functional connection (7) for the definition of gravitational constant:

$v_{G}=v_{p}=\sqrt{\frac{c^{5}}{G h}}=\sqrt{\frac{\left[0.299792458 \cdot 10^{9}\left(\frac{\mathrm{m}}{\mathrm{s}}\right)\right]^{5}}{6.67408 \cdot 10^{-11}\left(\frac{\mathrm{m}^{3}}{\mathrm{~kg} \cdot \mathrm{s}^{2}}\right) \cdot 6.626070040 \cdot 10^{-34}\left(\frac{\mathrm{kg} \cdot \mathrm{m}^{2}}{\mathrm{~s}}\right)}}=$

$=7.39994 \cdot 10^{42}\left(s^{-1}\right) \rightarrow 7.4 \cdot 10^{42}\left(s^{-1}\right)$.

$G=\frac{c^{5}}{v_{G}{ }^{2} h}=\frac{\left[0.299792458 \cdot 10^{9}\left(\frac{\mathrm{m}}{\mathrm{s}}\right)\right]^{5}}{\left[7.4 \cdot 10^{42}\left(\mathrm{~s}^{-1}\right)\right]^{2} 6.626070040 \cdot 10^{-34}\left(\frac{\mathrm{kg} \cdot \mathrm{m}^{2}}{\mathrm{~s}}\right)}=6.6739669698 \cdot 10^{-11}\left(\frac{\mathrm{m}^{3}}{\mathrm{~kg} \cdot \mathrm{s}^{2}}\right)$.

On the grounds of the found frequency (6) and known earlier wave laws [6], a wavelength of gravitational field $\lambda_{G}$ can be defined (8):

$$
\lambda_{G}=\frac{c}{v_{G}}=\frac{0.299792458 \cdot 10^{9}\left(\frac{m}{s}\right)}{7.4 \cdot 10^{42}\left(\mathrm{~s}^{-1}\right)}=4.051249|432| \cdot 10^{-35}(\mathrm{~m}) \text {. }
$$

Value $\lambda_{G}$ is confirmed with the numerical value of Planck's length $l_{p^{\prime}}$ yet, unlike it, the wavelength $\lambda_{G}$ has aexplicit physical sense [10].

There are no stable bounding planes at hand in elementary and circular quanta of space, as motion along them is impossible, and ribs, motion along which is possible, in the framework of Heisenberg uncertainty principle [11] have blurred configurations (spot),interpenetrating each other. The mutual penetration of such edges into each other provides a solid connection of quanta.

Taking into consideration the fact that in Quantum Mechanics a wave structure (photon) has also a structure of elementary particle [11], thus parameter $\lambda_{G}$ characterizes also sizes of an elementary particle, to which a hypothetical graviton [11] can be referred. Given that the gravitational field constitutes the basis of the Universe, and in the contemporary science the outer parameters object, which are lesser than value $\lambda_{G^{\prime}}$ are not proved by strict physical laws(except for a gravitational radius, which refers to the inner parameters object)that is why $\lambda_{G}$ characterizes a minimal possible size. That lets to bind it with minimal quanta of space of the Universe. And until new strict physical laws of the same level 
of significance as, (6)...(8), are found, which will provide the lesser numerical meaning of length, than value $\lambda_{G}(8)$, mainly it should be considered the minimally possible quantum physical size in the material world of the whole Universe.

Transition from 6 elementary quanta to the circular quantum of hexagonal shape goes indirectly with connection between Planck's constant $h$ and circular Planck's constant $\hbar(9)$, which are connected by number $2 \pi$ :

$$
\hbar=\frac{h}{2 \pi}=\frac{6.626070040 \cdot 10^{-34}(\mathrm{~J} \cdot \mathrm{s})}{2 \pi}=1.054571801 \cdot 10^{-34}(\mathrm{~J} \cdot \mathrm{s}) \text {. }
$$

This shows their role, though it is undefined, which of these constants $\hbar$ or $h$ should be named circular.

\section{Conclusion}

1. Parameters of all minimal quanta of space in the Universe are received by way of logical reasoning on the grounds of common principles of the material world structuring and scientific knowledge development from simple to the complicated, which do not contradict to the known laws of nature.

2. Minimal sizes of quantum of space are connected with wavelength $\lambda_{G}$ of the gravitational field, having the frequency $v_{G}=7.4 \cdot 10^{42} \mathrm{~s}^{-1}$ (constant Nastasenko): $\lambda_{G}=\frac{c}{v_{G}}=\frac{0.299792458 \cdot 10^{9}\left(\frac{m}{s}\right)}{7.4 \cdot 10^{42}\left(\mathrm{~s}^{-1}\right)}=4.051249|432| \cdot 10^{-35}(\mathrm{~m})$.

3. The connection of quanta of Universe space with wave parameters of the gravitational field is shown for the first time.

4. The condition of form minimization complies with elementary quanta in the shape of prisms with regular triangle basis and ribs length $\lambda_{G^{\prime}}$ which highlights their connection with quarks.

5. Circular grouping of 6 such kind elementary quanta in the shape, have of prisms form, contiguous to each other by lateral faces with universal central axes, forms complete quantum of space of the Universe in the shape of hexagonal prism its cyclic quantum space of University.

6. Quantum leap in elementary and circular quanta is possible along ribs only, and is impossible along diagonals in square and altitudes of triangle faces, because quant do not have stable limiting faces.

7. Ribs in quanta of space, in the frameworks of Heisenberg uncertainty principle, have blurred configurations (spot), interpenetrating each other for.

\section{References}

1. Vasilyeva VV, Krotovai AA, Bugay DV. History of Philosophy: Textbook for universities. M.: Academic Project: 2005.

2. Nastasenko VA. Methodology for solving creative tasks high level of complexity III. 2016; 4(48): 53-59.

3. Lenin VI. Materialism and empirio criticism. Political Literature Publishing House. 1976; 18: 277.

4. You are the only citizen of the world (Ukraine). The law of Ukraine. Kiev: School. 2003; 142.

5. Nastasenko VA. Ground of parameters minimum quantum of space of universe: Scientific Bulletin KhDMA. 2012; 1(6): 285-297.

6. Belov AF, Voskobounikov VG. Poly Liquid / Red. inch.: A.Yu. Echlin skies (gl. red.) typically, -3-e ed., pererabot. idop. -M.: Sov. Encyclopedia. 1989; 928: 638.

7. CODATA Internationally recommended values of the Fundamental Physical Constants. NIST reference.

8. Nastasenko VA. Possibly the most recent gravity Constantly settlement. Kiev, NTUU. 2013; 266-272.

9. Nastasenko VA. Possibility of Refining the Gravitational Constant and Solving the Task of Integrating the Gravitational and Electromagnetic Fields. Journal of Astrophysics \& Aerospace Technology. 2018; 6(1): 155. doi: 10.4172/23296542.1000155

10. Nastasenko VA. Union of gravitational and electromagnetic fields on the basis of nontraditional principles. ECIJ. 2017; 6(3-4): 19-30.

11. Alekseev DV, Bonch-Bruevich AM, Voronov-Romanov AS, et al. Physical encyclopedia hessky dictionary. Pod red. AM Prohorov (Ed.). Moskva :Sov. Encyclopedia.1983; 465: 826-33. 